\title{
ANALISIS DE LA BRECHA EN PLANEACION ESTRATEGICA DE TI ENTRE LAS INSTITUCIONES DE EDUCACION SUPERIOR DE NORTE DE SANTANDER Y EL MODELO IT4+
}

\author{
ANALYSIS OF THE GAP IN STRATEGIC PLANNING OF IT BETWEEN THE INSTITUTIONS OF \\ HIGHER EDUCATION OF NORTH OF SANTANDER AND THE MODEL IT4 +
}

\author{
MSc (c) Leidy Lisbeth Contreras Hernández, PhD. Torcoroma Velásquez Pérez, \\ PhD. Hugo Fernando Castro Silva
}

\author{
Universidad Francisco de Paula Santander, Sede Algodonal. \\ Ocaña, Norte de Santander, Colombia. \\ Tel.: 57-7-5690088 Ext. 182 \\ E-mail: 11contrerash@ufpso.edu.co; tvelasquezp@ufpso.edu.co; \\ hugofernando.castro@uptc.edu.co
}

\begin{abstract}
Resumen: Entendiendo la Planeación Estratégica como el proceso gerencial que desarrolla y mantiene una dirección estratégica para alinear las metas organizacionales con las oportunidades cambiantes, teniendo en cuenta las tecnologías de la información y las comunicaciones mediante la definición, implementación, ejecución, seguimiento y divulgación de un plan estratégico de tecnología y sistemas de información, mejor conocido como PETI; este principio enmarcado dentro de Gobierno Corporativo. El Gobierno corporativo de TI entendido como un conjunto de responsabilidades y prácticas con el fin de proveer direccionamiento estratégico, el marco de Gobierno corporativo está orientado a alcanzar los objetivos de la entidad, que se puede clasificar en cuatro categorías la Estrategia, las Operaciones, la Información y el Cumplimiento. El presente documento tiene como fin el análisis de la brecha en planeación estratégica de TI entre las Instituciones de Educación Superior de Norte de Santander y el modelo IT4 + propuesto por el Ministerio de las Tecnologías de la Información y la Comunicación en Colombia. Para el desarrollo del trabajo se utiliza una metodología descriptiva la cual permiten a través de estos estándares, identificar los elementos necesarios para realizar dicho análisis.
\end{abstract}

Palabras clave: Gobierno de TI, PETI, COBIT 5.0, ISO 38500, Modelo IT4+.

\begin{abstract}
Understanding Strategic Planning as the managerial process that maintains and maintains a strategic direction for organizational goals with changing opportunities, taking into account information and communication technologies through the definition, implementation, execution, monitoring and dissemination of a Technology strategies and information systems, better known as PETI; This principle is framed within the Corporate Government. The corporate governance of IT has become a set of responsibilities and practices in order to provide a strategic direction, the framework has been focused on the objectives of the entity. Information and compliance. The purpose of this document is to analyze the gap in strategic IT planning between the Higher Education Institutions of Norte de Santander and the IT4 + model provided by the Ministry of Information and Communication Technologies in Colombia. For the development of the work, a
\end{abstract}


descriptive methodology is used, which is obtained through these levels, identifying the necessary elements to perform said analysis.

Keywords: IT Governance, information systems \& technology (IST's) strategic plan, COBIT 5, ISO/IEC 38500, Model IT4+.

\section{INTRODUCCIÓN}

La incorporación de tecnología en la ejecución de los procesos de las organizaciones debe realizarse con base a una planificación corporativa con visión y objetivos claros, alineada a los objetivos institucionales $\mathrm{y}$ con un enfoque de soporte efectivo a sus procesos, apoyada en modelos validados y alineados con la normatividad vigente en el país.

El Gobierno de las TI es el sistema a través del cual se dirige y controla la utilización de las TI actuales y futuras. Supone la dirección y evaluación de los planes de utilización de las TI que den soporte a la organización y la monitorización de dicho uso para alcanzar lo establecido en los planes de la organización. Incluye las estrategias y políticas de uso de las TI dentro de la organización (ISO 38500 , 2008). La planeación estratégica de Tecnologías de la Información apoya el gobierno corporativo de TI.

\subsection{OBJETIVOS}

En ese sentido la presente investigación está orientada a analizar la brecha en planeación estratégica de TI entre las Instituciones de Educación superior de Norte de Santander y el modelo IT4+ estableciendo un marco de referencia para evaluar el nivel de madurez en la planeación Estratégica de Tecnologías de la Información determinando las causas más relevantes que dan lugar a dicha brecha y el establecimiento de indicadores que permitan la alineación con el modelo de gestión IT4+ del Ministerio de las Tecnologías de la Información y Comunicación.

La planeación estratégica es una actividad planeada que se centra en el éxito y la mejora. (Rochetti, 2006) Se asume la planificación estratégica como reto, proceso en marcha y espejo de la visión, misión y valor de la organización. (Pirtea, Nicolescu, \& Botoc, 2009) Es una manera de definir las metas y objetivos que según la Fundación para la investigación (2014), se requiere de las personas para confirmar el resultado que cumple el objetivo
(Rashida, y otros, 2016). Consideran el medio ambiente externo e interno como parte de la planeación estratégica (Hannah, Balthazard, Waldman, Jennings, \& Thatcher, 2013). En (Jiménez, 1982) es definida como un proceso de toma de decisiones para alcanzar el futuro deseado (Jiménez, 1982) ; así mismo (Kotler, 1990) indica que es un proceso gerencial de desarrollar y mantener una dirección estratégica que pueda alinear las metas y recursos con sus oportunidades cambiantes (Kotler, 1990). Dentro de los objetivos de fortalecimiento institucional, se debe liderar la gestión estratégica con tecnologías de la información y las comunicaciones mediante la definición, implementación, ejecución, seguimiento y divulgación de un plan estratégico de tecnología y sistemas de información PETI (Ministerio de Las tecnologias de la Informacion, 2016). Si se definen y se tiene clara la misión de las empresas, les será más fácil a los gerentes imprimirles dirección y propósito y responderán positivamente a los cambios del entorno (Rodríguez Valencia, 2016). Los catalizadores de los procesos organizacionales son las tecnologías de la información pues sin lugar a dudas se constituyen en herramientas de apoyo a la gestión empresarial, apalancando la construcción de estrategias orientadas a la competitividad y la innovación, generando así sostenibilidad para la organización y la sociedad (Stern, 2002).

La planeación Estratégica de Tecnología de la Información (PETI) influye en la ventaja competitiva de las Instituciones de educación superior (Information Systems Department, Bina Nusantara University, 2016). Algunas compañías de Fortune 500 y agencias gubernamentales han validado los retornos de la inversión del $700 \%$ resultado de su inversión en PETI (Cannon, 2016), esto ha impulsado a grandes universidades que adoptaran esta tendencia, la University of Oxford ha implementado un Plan Estratégico que la prepara para lograr el máximo beneficio de las innovaciones en tecnología de la información (The IT Strategic Plan Oxford, 2013) (Oxford, 2013). La Universidad de Harvard mediante su plan estratégico de TI lanzó varias iniciativas 
estratégicas como lo es Harvard Phone, Cloud y Devops, HarvardKey entre otras, las cuales transforman la manera de trabajar, enseñar y aprender en Harvard (Harvard, 2015) (University, 2015). Así mismo, el gobierno colombiano incursiona en esta área a través del Ministerio de las TIC liderando el modelo IT4+ el cual está alineado con la estrategia empresarial y permite desarrollar una gestión de TI que genere valor estratégico para la organización y sus clientes conectando la Estrategia con el Negocio y la Gestión de TI (Ministerio de Las tecnologias de la Informacion, 2016).

\subsection{ESTADO DEL ARTE}

En (Kaminski, 2000) se habla del cambio en las organizaciones, apoyado en el estudio (Kaminski, 2000) se identifica las habilidades de liderazgo como elementos fundamentales en la planeación estratégica de las universidades. Las universidades colombianas han dado sus pasos sobre este tema (Universidad Nacional de Colombia, 2016), (Universidad de Pamplona, 2012), (Universidad Simon Bolivar, 2013), (Universidad de Santander UDES, 2013), (Universidad Francisco de Paula Santander, 2011), (Sanguino, Lobo, \& Arrieta, 2015) la experiencia y conocimiento de las instituciones y las personas que la conforman son fundamentales en el mejoramiento y la resolución de problemas; de tal manera, se hace necesario adoptar algunas rupturas estratégicas en términos de la gestión de TI (Ministerio de las Tecnologias de la Información, 2017). Se observa que las Instituciones de educación superior más relevantes de Norte de Santander poseen dentro de sus objetivos institucionales y misionales la incorporación de tecnología; sin embargo, a pesar que el Ministerio de las TIC propone el modelo IT4+ para PETI, estas organizaciones no siguen dichos lineamientos ni son implementados. Lo anterior se evidencia en el poco control para la adquisición, el uso y la administración de los recursos de TI y la no existencia de un marco de referencia que evalúe los niveles de madurez en el que se encuentran.

La llegada de la era de la economía del conocimiento hace que la tecnología de la información se convierta en un arma competitiva eficaz que puede ayudar a una empresa a sobrevivir en la sociedad moderna y obtener un mayor desarrollo (Yujie \& Wang, 2010). En Colombia son cada vez más las instituciones de educación superior que hacen uso de las herramientas TIC como herramienta para la administración de sus procesos y recursos educativos (Puello, Cabarcas, \& Martelo, 2013). Desde 2007, el Ministerio de Educación Nacional materializó el proyecto Plan Es TIC con el propósito de impulsar procesos de planeación estratégica para la incorporación de las TIC en los procesos educativos de las IES colombianas (Ministerio de Educacion Nacional, 2012). Actualmente existe un número significativo de instituciones que ya cuentan con su plan estratégico, presentándose un escenario que permite empezar a intercambiar intereses, experiencias y proyectos, de manera que las IES involucradas se vean beneficiadas y que como producto de esto, el tema de incorporación de TIC en procesos educativos también se vea fortalecido a nivel nacional. (Colombia Aprende, 2009) La planeación estratégica de TI es indispensable para las instituciones de Educación Superior pues permite la preservación, y aseguramiento de la información siendo esta un activo valioso que requiere contar con estrategias de alto nivel que permitan el control y administración efectiva de los datos (Universidad distrital, 2016); decidiendo la capacidad tecnológica, adoptando y adaptando la tecnología, creando capacidades tecnológicas en las IES que permitan innovar en productos y servicios para ser más competitivas (Agudelo , Niebles, \& Gallon, 2005).

A nivel mundial existen instituciones educativas que se caracterizan por su liderazgo en la aplicación de tecnologías, como es el caso de la Universidad de Stanford, el Instituto Tecnológico de Massachusetts (MIT), la Escuela Politécnica Federal de Zúrich (EPFZ) de Suiza, y la Universidad de Cambridge; ahondan en la manera de aprender de sus alumnos, la incidencia de la tecnología en el proceso y la adaptación que necesitan los profesores a las innovaciones (Cano, Couto, Barbara, \& Cristina F., 2014). Las universidades colombianas han recibido apoyo de universidades estadounidenses líderes en Transferencia de Tecnología (Arkansas, Connecticut, Misuri, Colorado, la Universidad del Sur de la Florida y la Universidad de Florida Central) con el objetivo de convertir sus investigaciones científicas exitosas, en empresas con potencial de crecimiento rápido, rentable y sostenido (Martin, 2013). El gobierno colombiano a través del Ministerio de las TIC sugiere que se soporte adecuadamente los procesos misionales de las organizaciones, potenciando la base tecnológica y de comunicaciones que apalanque la transformación y la estrategia de las instituciones en los diferentes sectores (Ministerio de Las Tecnologias de la Informacion, 2016). En Norte de 
Santander según el plan de Desarrollo de Norte de Santander (2016-2019) su objetivo es cerrar la brecha digital en el departamento a través del fortalecimiento del ecosistema digital en infraestructura tecnológica fijando una meta esperada para el año 2019 del $100 \%$ en Implementación PETI en las instituciones públicas de Norte de Santander. Teniendo en cuenta estos lineamientos y herramientas que proporcionan las entidades gubernamentales; a las organizaciones se les dificulta implementar dichas estrategias ya que desconocen su nivel de madurez tecnológico y no poseen políticas internas para ayudar a los trabajadores a adaptarse a medida que se agregan nuevas tecnologías (Fernandez, 2017), las cuales son puntos de partida para reducir la brecha existente y facilitar la implementación del Modelo IT4+ propuesto por Min TIC.

Michael Dooris, Director de Investigación sobre Planeación del Centro de Calidad y Planeación en The Pennsylvania State University, plantea un desarrollo progresivo y cada vez más maduro del sentido de la planeación estratégica, en el contexto de la educación superior (Cifuentes, 2006), de igual forma hay un renovado interés en la planeación estratégica de TI, dirigida en parte por los nuevos retos que afrontan las instituciones de educación superior, junto con la creciente toma de conciencia de que TI debe ser parte de la solución a estos retos. Dado lo anterior la planeación estratégica de TI se ha venido afianzando en las grandes universidades a nivel mundial como eje central para el desarrollo económico y sostenible. Las mejores universidades del mundo entre ellas, Massachusetts Institute of Technology (MIT), Stanford University, Harvard University, California Institute of Technology (Caltech), University of Cambridge, University of Oxford, UCL (University College London), la Imperial College London entre otras cuentan con una sólida Planeación estratégica de TI orientada al desarrollo tecnológico, fortaleciendo sus procesos y contribuyendo en avances tecnológicos como lo menciona la prestigiosa revista Technology Review, editada por el Instituto Tecnológico de Massachusetts (MIT) los 10 avances científicos y tecnológicos que prometen revolucionar el mundo entre ellas herramientas para revertir la parálisis, un completo mapa de las células humanas y computadores cuánticos son algunos de los avances que se verán en los próximos años "Todas estas tecnologías tienen el poder de trascender. Afectarán la economía y nuestra política, mejorarán la medicina o influirán en nuestra cultura por tanto hay que conocerlas ahora".
(Ministerio de las Tecnologias de la Información, 2017).

\section{METODOLOGÍA}

Teniendo en cuenta el propósito de la presente investigación, de analizar la brecha en planeación estratégica de TI entre las Instituciones de Educación superior de Norte de Santander y el modelo IT4+, se llevará a cabo una investigación cuantitativa, ya que de acuerdo con (Garcia M. L., 1995) para que exista metodología cuantitativa debe haber claridad entre los elementos de investigación desde donde esta inicia hasta donde termina, abordando los datos de manera estática y asignándole un significado numérico a través de la estadística, con la finalidad de hacer inferencias y de acuerdo con Tamayo (Tamayo, El Proceso de la Investigación Científica, 2007), la metodología cuantitativa consiste en el contraste de teorías ya existentes a partir de una serie de hipótesis surgidas de la misma, siendo necesario obtener una muestra, ya sea en forma aleatoria o discriminada, pero representativa de una población o fenómeno objeto de estudio. Del mismo modo se desarrolla una investigación descriptiva "La investigación descriptiva busca especificar propiedades, características y rasgos importantes de cualquier fenómeno que se analice" (Hernández, Fernández \& Baptista, 2003) (Hernández, R., Fernández, C., \& Baptista, P. , 2003) y Según (Fidias, 2012) dicha investigación consiste en la caracterización de un hecho, fenómeno, individuo o grupo, con el fin de establecer su estructura o comportamiento. Los resultados de este tipo de investigación se ubican en un nivel intermedio en cuanto a la profundidad de los conocimientos se refiere.

Para la presente investigación se toma como población las Instituciones de Educación Superior de Norte de Santander con mayor número de estudiantes matriculados en el Departamento según Sistema Nacional de Información de la Educación Superior (SNIES-2016) las cuales son Universidad de Pamplona -UNIPAMPLONA ubicada en la ciudad de Pamplona de carácter público, Universidad Francisco de Paula Santander - UFPS ubicada en la ciudad de Cúcuta y seccional en Ocaña de carácter público, Universidad Simón Bolívar extensión Cúcuta - UNISIMON de carácter Privado, Universidad de Santander extensión Cúcuta-UDES de carácter privado. La muestra que se determina para la presente investigación son los líderes de los procesos del área tecnológica de cada una de las Instituciones de Educación superior de 
Norte de Santander definidas como población anteriormente.

\section{RESULTADOS}

\subsection{Nivel de Madurez}

Para evaluar el nivel de madurez se toma como referencia el modelo de madurez capability maturity model (CMM por sus siglas en inglés) el cual describe por cada uno de sus cinco niveles, inicial,

repetible, definido, gerenciado y optimizado, cuáles son las características que marcan la evolución de estos procesos, y las áreas claves en las que deben implementarse un conjunto de prácticas o focalizarse los cambios en aras de consolidar en este caso la alineación de los procesos con las tecnologías de la información; y los niveles de madurez de gestión de TI de Gartner funcional, habilitador, contributivo, diferenciador y transformador para llevar a cabo la adaptación a la realidad de la gestión de TI en el sector público.

\section{Tabla 1 Valoración Niveles de Madurez.}

\begin{tabular}{c|c} 
NIVEL DE MADUREZ & VALOR \\
\hline Inicial & 1 \\
\hline Consiente & 2 \\
\hline Definido & 3 \\
\hline Cuantitativamente gestionado & 4 \\
\hline Optimizado & 5
\end{tabular}

Para evaluar el nivel de madurez se elaboró un instrumento con 32 preguntas las cuales hacen parte de las cuatro fases de planeación estratégica de tecnología de la información teniendo como base la valoración de 1 a 5 como se evidencia en la tabla 1 y su aplicación se realizó a los líderes de tecnología de las siguientes Universidades

- Universidad de Pamplona

- Universidad Simón Bolívar Seccional Cúcuta

- Universidad de Santander seccional Cúcuta

- Universidad Francisco de Paula Santander

Nivel Madurez Global

Figura 1 Nivel de Madurez Global por fasos do nlanoarión Fstratóoira do TI

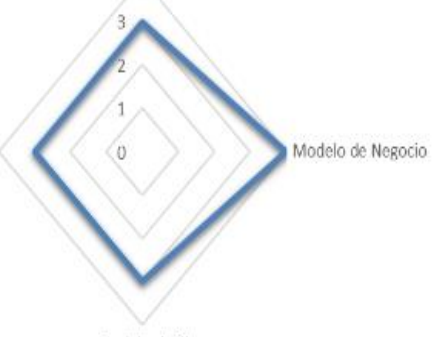

seccional Ocaña

En la Figura 1 tipo radar se evidencia el nivel de madurez global por fases de planeación estrategia de TI de las Instituciones de educación superior de Norte de Santander evaluadas en donde la fase que posee mayor madurez es el modelo de negocio con una valoración de 4 (cuantitativamente gestionado), lo que significa que las iniciativas de modelo operativo están bien establecidas en las instituciones siendo un diferenciador con las otras fases.

Así mismo las fases de análisis situacional, modelo de TI y Modelo de Planeación cuentan con una madurez de 3 indicando que las IES de Norte de Santander tienen definida la planeación estratégica de TI parcialmente, sin cuantificación ni mejora continua.

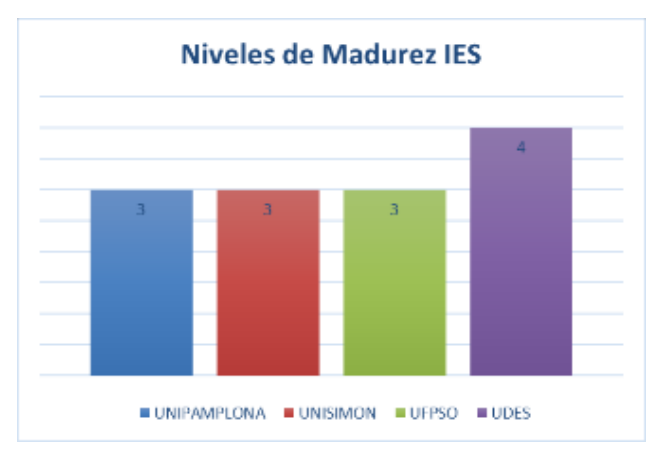

Figura 2. Nivel de Madurez IES Norte de Santander en Planeación Estratégica de TI

en la figura 2 se aprecia el nivel de madurez en el cual se encuentra cada una de las instituciones de educación superior de Norte de Santander, donde se evidencia que la universidad de Santander sede Cúcuta es quien posee un nivel de madurez más alto con una valoración de 4 (Cuantitativamente gestionado), indicando que esta institución tiene plenamente establecidas iniciativas de planeación estratégica de tecnologías de la información evidenciándose en la perfecta integración de la tecnología con la arquitectura de procesos.

\subsection{Análisis de la brecha}

3.2.1 Causas relevantes que determina la brecha entre las IES y el Modelo It4+ 
Confundir estrategia con planeación: La Estrategia tiene que ver con la definición de las ventajas competitivas de la empresa, la creación de valor para el cliente y la diferenciación que busca la compañía en el mercado mientras que planeación tiene que ver con el proceso de formular planes y objetivos.

Llevar a cabo actividades de planeación estratégica sin una metodología adecuada.: Se requiere de una junta directiva sólida con conocimiento y experiencia que aporte valor a la hora de tomar decisiones y que ayude a resolver los problemas propios de cada organización.

Proceso de formulación estratégica desligado del proceso de revisión estratégica: Mucho activismo de la alta dirección en la construcción del plan y poco liderazgo para hacerle seguimiento, monitorear y ajustar los resultados de la implementación estratégica.

Sistemas obsoletos: Uso de métodos obsoletos y torpes para llevar a cabo los objetivos deseados, por tanto la productividad disminuye significativamente.

Falta de compromiso desde la gestión empresarial con los procesos de TI: Las juntas empresariales, los ejecutivos y la gerencia tienen que adoptar la TI como cualquier otra parte importante del negocio, teniendo claro que no es un método antojadizo ni debe ser por regulación, sino por un sentido común de hacer las cosas bajo una perspectiva de mejores prácticas y con el objetivo de generar valor a las diferentes partes interesadas de la organización.

La falta de equilibrio y alineación de los elementos clave empresariales: tecnología, procesos de negocios y personas: los recursos tanto tecnológicos, humanos como financieros deben optimizarse, pues el riesgo se puede identificar y controlar; garantizando que los objetivos organizacionales son alcanzados.

Falta de interoperabilidad: Se debe fortalecer los esquemas de interoperabilidad que estandaricen y faciliten el intercambio de información con las áreas y entes externos, el manejo de fuentes únicas de información y la habilitación de servicios.

Adquisición de tecnología sin realizar un análisis de necesidades reales que contribuyan al cumplimiento de los objetivos misionales de la organización: Los sistemas de información se deben definir teniendo en cuenta la cadena de valor de la entidad con sus procesos de planeación, misionales y de apoyo.

Falta de Alianzas estratégicas tecnológicas: Se evidencia falta de liderazgo en los directivos de las instituciones, para concretar socios estratégicos en tecnología.

Recurso humano no capacitado: Los encargados del reclutamiento deben tener habilidades para detectar a las personas adecuadas para cada posición, limitando así el riesgo de equivocarse.

Arquitectura de Información sin establecer: Información desorganizada dificultando su comprensión y asimilación, así como las tareas que ejecutan los usuarios en un espacio de información definido.

No se encuentra una cultura y gobierno de TI establecido: No existe alineación de las Tecnologías de la información y la comunicación (TI) con la estrategia del negocio. la organización de TI debe ser responsable de todo lo relacionado con su área de acción, desde la gestión de relaciones con los clientes de tecnologías de información y la planificación de servicios hasta la prestación de servicios, incluso si externaliza algunas de sus operaciones.

La exclusión del responsable de TI de la institución en la planeación estratégica. El personal de TI es quien conoce las tendencias en tecnologías que pueden soportar y apoyar las necesidades que presentan las diferentes áreas de las organizaciones o instituciones, sin embargo no se tiene en cuenta para la toma de decisiones en temas relacionados con su área.

No existen acuerdos de niveles de servicio: $\mathrm{Se}$ deben definir las necesidades de la institución controlando las expectativas de servicio en relación a la capacidad del proveedor, para proporcionar un marco de entendimiento, simplicidad de asuntos complicados, la reducción de las áreas de conflicto y favorecer el diálogo ante la disputa.

Sistemas de Información insuficientes. Los sistemas de información de Apoyo, misionales y de direccionamiento estratégico no son suficientes para soportar los objetivos misionales de la institución.

Conectividad deficiente: Perdida de información, Congestión de la red, interferencias, cableado defectuoso, Se requiere de una renovación 
tecnológica que soporte los requerimientos de todas las áreas de la institución.

Las estrategias de Uso y Apropiación de Tecnología no son suficientes: Se debe vincular a

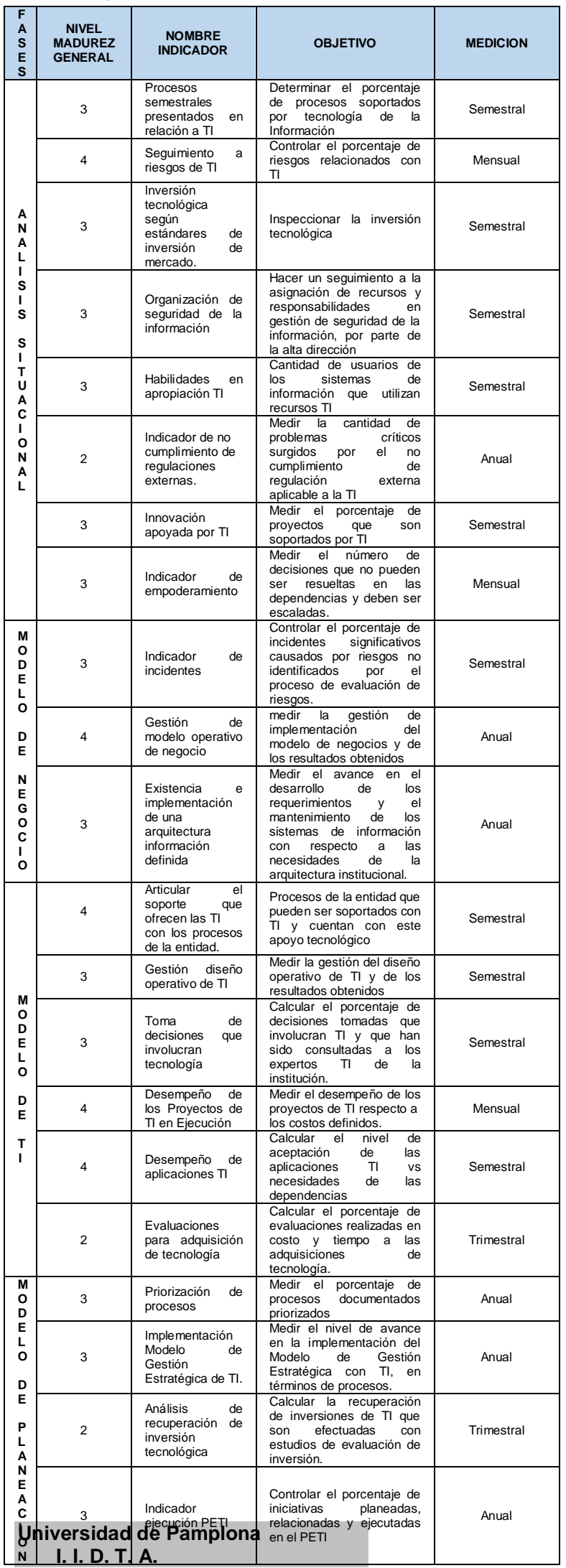

las personas $y$ desarrollar una cultura o comportamientos culturales que faciliten la adopción de tecnología para que las inversiones en TI sean productivas; para ello se requiere realizar actividades de fomento que logren un mayor nivel de uso y apropiación.

Se evidencian lineamientos de planeación sin implementar: Dichos lineamientos se encuentran documentados sin embargo no se tienen en cuenta para su ejecución.

No se tienen indicadores para Planeación estratégica de TI: Se deben definir los indicadores de avance e impacto de la estrategia de TI

No se tiene un análisis financiero de la planeación estratégica de TI: Se debe realizar un análisis financiero de la ejecución del plan.

\subsubsection{Indicadores para la alineación de PETI} IES vs modelo IT4+

\section{Tabla 2. Indicadores para disminuir la Brecha}

Una vez definidas las causas de cada uno de las fases de planeación estrategia de TI que da a lugar a la brecha existente entre las instituciones de educación superior de norte de Santander y el modelo IT4+ se plantean indicadores que permiten su alineación con base en los subcomponentes evaluados que obtuvieron un nivel de madurez global inferior e igual a 4 como se puede evidenciar en la tabla 2

\section{CONCLUSIONES}

A nivel nacional se observan lineamientos del gobierno Colombiano en cuanto a planeación estratégica de Gestión de la Tecnología de información, a través del Ministerio de Tecnologías de la Información lideran una propuesta para el sector público de la estructura que deben tener los Planes estratégicos de Tecnologías de la Información basado en el modelo IT4+ que conecta la Estrategia con el Negocio y la Gestión de TI, a través de sus dimensiones: Estrategia de TI, Gestión de TI, Gobierno de TI, Información, Sistemas de Información, Servicios Tecnológicos y del Uso y Apropiación (Ministerio de Las Tecnologias de la Informacion, 2016). En el ámbito de las Instituciones de Educación Superior la mejor Universidad de Colombia (Ranking de Universidades Colombianas - Revista Dinero, 
2017) La Universidad Nacional de Colombia través de la Dirección Nacional de Tecnologías de la Información y Comunicaciones DNTIC y en concordancia con el programa de gobierno y las necesidades instituciones tendientes al plan global de desarrollo 2016-2018 poseen un plan estratégico de Tecnología V.1.0 actualizado (Actualización PETI Universidad Nacional de Colombia, 2016).

A nivel regional al revisar aspectos generales de la planeación estratégica de TI en el departamento de Norte de Santander y teniendo en cuenta las Universidades con mayor número de estudiantes Según el Sistema Nacional de Información de La Educación Superior (SNIES-2016) en Norte de Santander se observa que; La universidad de Pamplona con 43.571 estudiantes matriculados, dentro de sus servicios de consultoría PLATAFORMA hacen mención de la planeación estratégica de Tecnologías de la Información (CIADTI, 2016) (CIADTI, 2016). La Universidad Francisco de Paula Santander con 48.954 estudiantes matriculados, se diseñó una propuesta de Planeación Estratégica de Tecnologías de la Información denominado Diseño De Un Plan Estratégico De Tecnologías De Información Para La Universidad Francisco De Paula Santander Ocaña (Sanguino, Lobo, \& Arrieta, 2015).

De otra parte, la Universidad Simón Bolívar seccional Cúcuta con 8665 estudiantes matriculados; dentro de su plan estratégico de Desarrollo (2013-2017) contempla la Consolidación del uso y apropiación de las tecnologías de la información y la comunicación en los procesos académicos y administrativos, pero no se evidencia una planeación Estratégica de Tecnologías de la Información. Del mismo modo se tiene que la Universidad de Santander UDES con 4.963 estudiantes Matriculados, en su Plan de Desarrollo (2013-2018) uno de los ejes estratégicos de desarrollo es la incorporación de Tecnologías de la Información, donde ha fortalecido su infraestructura física, tecnológica y logística para garantizar el ejercicio de la docencia, la investigación y el bienestar. La Universidad de Santander posee una infraestructura tecnológica (audiovisuales, conectividad, equipos de laboratorio) dedicados a la docencia como un aporte significativo al mejoramiento de la calidad de la educación, además de sus laboratorios, espacios de estudios y trabajo individual o en grupo, recursos bibliográficos (Bases de datos, publicaciones, entre otros), tanto en soporte físico como digital.
En Norte de Santander según el plan de Desarrollo de Norte de Santander "Un Norte Productivo para Todos" (2016-2019) en su eje temático competitividad e innovación a través de las TIC cuyo objetivo es cerrar la brecha digital en el departamento a través del fortalecimiento del ecosistema digital en infraestructura tecnológica, servicios, aplicaciones, al observar que para el año 2015 la proporción de la implementación de planes estratégicos de Tecnología de la Información es del 0\%, se fija una meta esperada para el año 2019 del $100 \%$ en Implementación PETI en las instituciones de Norte de Santander.

Una vez evaluado el nivel de madurez se encontró que la universidad de Santander sede Cúcuta es quien posee un nivel de madurez más alto con una valoración de 4 (Cuantitativamente gestionado), indicando que esta institución tiene plenamente establecidas iniciativas de planeación estratégica de tecnologías de la información evidenciándose en la perfecta integración de la tecnología con la arquitectura de contenidos.

En cuanto a la universidad Francisco de Paula Santander sede Ocaña, Universidad de Pamplona, Universidad simón Bolívar sede Cúcuta arrojaron un resultado de madurez de 3 (Definido) lo cual indica que estas instituciones han puesto en marcha las iniciativas de planeación estratégica de TI sin embargo se tiene una infraestructura y estrategia básica de TI

\section{BIBLIOGRAFIA}

Alvernia Acevedo, S., \& Rico Bautista, D. (2017). Análisis de una red en un entorno IPV6: una mirada desde las intrusiones de red y el modelo TCP/IP. REVISTA COLOMBIANA DE TECNOLOGÍAS DE AVANZADA, 1(29).

Arango , M. S., Londoño , J. S., \& Zapata, J. C. (Enero/Junio de 2010). Arquitectura Empresarial- Una vision general. Revista Ingenierías Universidad de Medellín, 9(16), 101-111.

Agudelo , E., Niebles, L., \& Gallon, L. (2005). La gestión tecnológica como herramienta de planeación estratégica y operativa para las unidades de información. 28(2), 89-114.

Arboleda, H., Lucumí, W. S., \& Torres, A. A. (01 de Octubre de 2015). Modelo de Gestión y Gobierno de Tecnologías de Información en universidades de 
Colombia: Caso Instituciones de Educación Superior en el Departamento del Cauca. Cauca, Colombia.

Arévalo, J. B. (2015). El problema de la brecha tecnológica: Un asunto de cultura. Sinapsis, 35-43.

BEMTUR. (November de 2015). 3rd Global Conference on Business, Economics, Management and Tourism,. Rome, Italy.

Bonfante, M., \& Castillo, A. (2014). Integración de sistema multi-agente, ontologías y procesos de negocios como marco tecnológico de la estrategia "gobierno en línea". REVISTA COLOMBIANA DE TECNOLOGÍAS DE AVANZADA, $1(23)$.

Cannon, M. Q. (05 de Mayo de 2016). The Importance of IT Strategic Planning and How to Approach It. EE. UU.

Cano, R. J., Couto, R. C., B. C., \& C. P. (03 de Octubre de 2014). Las universidades más punteras del mundo en el uso de las tecnologías. San Francisco, EE.UU.

Cassidy, A. (1998). A Practical Guide to Information Systems Strategic Planning. Florida: Auerbach Publications.

Chiavenato, I. S. (2017). Planeación estratégica: fundamentos y aplicaciones. Distrito Federal, MX : McGraw-Hill Interamericana .

CIADTI. (2016). Centro de Investigacion Aplicada y Desarrollo en Tecnologias de la informacion. Obtenido de www.ciadti.com.co

Cifuentes, J. (2006). Experiencias de Planeación en la Pontificia Universidad Javeriana. Seminario Internacional "Dirección Estratégica Universitaria”. Barcelona.

Colombia Aprende. (2009). Planes TIC en las IES colombianas. Colombia.

CRUE. (2016). Gobierno de Tecnologias de las tecnologias de la informacion en Universidades. Conferencia de Rectores de las Universidades Españolas, (pág. 9). Madrid.

De la Cruz, A., \& Mauricio, D. (2007). Una Revisión de la Gestión de Servicios de Tecnologías de Información. Revista de investigación de Sistemas e Informática UNMSN, 4(1).

De la Cruz, A., \& Mauricio, D. (2007). Una Revisión de la Gestión de Servicios de Tecnologías de Información. Revista de investigación de Sistemas e Informática UNMSN, 4(1).
Departamento Administrativo de la Funcion Publica. (07 de Marzo de 2016). Decreto 415 de 2016. Articulo 2.2.35.3. Bogotá, Colombia.

Druker, P. (1954). The Practice of Management. Estados Unidos: Harper \& Brothers publishers.

Fernandez, K. (2017). ¿Conoce el nivel de madurez tecnológica que tiene en su empresa? ITNOW.

Fidias, G. A. (2012). El Proyecto de Investigación, Introduccion a la metodología Científica. Caracas, República Bolivariana de Venezuela: EPISTEME, C.A.

Garcia, M. L. (1995). Proyecto de Investigacion.

Garcia, R. G. (2017). El método Delphi para el procesamiento de los resultados de encuestas a expertos o usuarios en estudios de mercado y en la investigación educacional. Universidad Simón Bolivar.

Garrido, S. B. (2006). Direccion Estratégica. España: Mc Graw Hill.

Gobernacion Norte de Santander. (2016). Plan de Desarrollo de Norte de Santander"Un Norte Productivo para Todos" (20162019). Cúcuta.

Hannah, S. T., Balthazard, P. A., Waldman, D. A., Jennings, P. L., \& Thatcher, R. W. (2013). The psychological and neurological bases of leader self-complexity and effects on adaptive decision-making. Journal of Applied Psychology, 98(3), 393-411.

Harvard University. (Abril de 2015). CIO Council Updated IT Strategic Plan. Cambridge, Massachusetts, EE. UU.: Harvard University.

Hernández, R., Fernández, C., \& Baptista, P. . (2003). Metodología de la Investigacion. Mexico: Mc Graw Hill.

Information Systems Department, Bina Nusantara University. (2016). Influence of IS adoption and IS capability to IS innovation and IS strategic planning and its implications to competitive advantage of private higher education institution. 7883. Bandung, Indonesia: IEEE Conference Publications.

ISACA. (2010). Gobierno de las TIC ISO/IEC 38500 (Vol. 1). Isaca Journal.

Jiménez, W. (1982). Introducción al Estudio de la Teoría Administrativa. (Vol. Editorial FCE.). Mexico: Editorial FCE.

Jones, J. B. (2013). A Strategic Informatics Approach. labmed.theclinics.com, Science Direct, 33(1), 161-181. 
Juiz, C., Gomez, M., \& Barceló, M. (2012). Business/IT Projects Alignment through the Project Portfolio, Approval Process as IT Governance Instrument. Procedia Social and Behavioral Sciences, 10-75.

Kaminski, G. (2000). The Causes of Banking and Balance of Payments Problems. American Economic Review, 473-500.

Kotler, P. y. (1990). Mercadeo de servicios profesionales. Santafé de Bogotá:: Ed. Legis.

Landeta, J. (2002). El Metodo Delphi. Barcelona: Ariel.

Lankhorst, M. (2005). Enterprise Architecture at Work -Modeling,. 352 p.

Listone, H., \& Turoff, M. . (2002). he Delphi method, techniques and applications. Addison wesley publishing.

Luis Omar Tangarife Tellez, M. P. S. Delgado, W. M. R.Contreras. (2014). Modelo de interventoría de tecnologías de información en el área de conocimiento de la gestión del alcance de pmbok® y alineado con iso 21500 y cobit $\AA$. REVISTA COLOMBIANA DE TECNOLOGÍAS DE AVANZADA, ISSN: 1692-7257. 1(23).

M. C. Bonfante, A. Castillo. (2014). Integración de sistema multi-agente, ontologías y procesos de negocios como marco tecnológico de la estrategia "gobierno en línea". REVISTA COLOMBIANA DE TECNOLOGÍAS DE AVANZADA, ISSN: 1692-7257. 1(23).

Martin, J. (01 de Marzo de 2013). 6 universidades estadounidenses apoyan a Colombia en Transferencia de Tecnología.

Martinez, D. P., \& Milla, A. G. (2012). Mapas estratégicos. En D. P. Martinez, \& A. G. Milla, La elaboracion del plan estrategico y su implantacion a traves del cuadro de mando integral (pág. 25). Madrid, España: Ediciones Díaz de Santos.

Mendez, C. E. (2011). Diseño y Desarrollo del Proceso de Investigacion con enfasis en ciencias empresariales. Mexico: Limusa.

Ministerio de Educacion Nacional. (28 de Mayo de 2012). Universidades lideran la planeación estratégica en la incorporación de TIC. Bogotá.

Ministerio de Las tecnologias de la Informacion. (2016). G.ES.06 Guía Cómo Estructurar el Plan Estratégico de Tecnologías de la Información - PETI. Bogotá D.C: version 1.0 .
Ministerio de Las Tecnologias de la Informacion. (2016). Version actualizada del modelo de Gestion IT4+. Bogotá D.C.

Ministerio de las Tecnologias de la Información. (01 de 05 de 2017). MINTIC. Recuperado el 29 de 09 de 2017, de http://www.mintic.gov.co/portal/604/w3article-7967.html

Mintzberg, H., \& Quinn, J. (1998). El proceso estratégico, conceptos y casos. Mexico: Prentice Hall.

Osorio, J. A. (2011). Hacia la Planeación Estratégica en Tecnologías de la nformación: Definiciones y Modelos. Fundación Universitara Calotica del Norte, 13.

Oxford, U. o. (2013). The IT Strategic Plan. . Oxford, Reino Unido.

Oxford, University of. (2013). The IT Strategic Plan. Oxford, Reino Unido.

Padilla, D. R., \& Garza, M. C. (1997). Empresas competitivas : una estrategia de cambio para el éxito. mexico: McGraw Hill.

Perez, T. V. (s.f.). Gobernabilidad de TI en las Empresas Colombianas. Mérida, Venezuela: Universidad de los Andes.

Perez, T. V., Velasquez, A. P., \& Perez, Y. P. (s.f.). Un enfoque de buenas practicas de gobierno corporativo de TI. Tecnura: Tecnologia y cultura afirmando el conocimiento, 19(1), 159-169.

Pino, R. M. (2009). Que es la brecha digital o tecnologica. Universidad ICESI.

Pirtea, M., Nicolescu, C., \& Botoc, C. (2009). The role of strategic planning. Annales Universitatis Apulensis Series Oeconomica, 11(2), 953-957.

Puello, P., Cabarcas, A., \& Martelo, R. (2013). Sistema de Información Gerencial para la Administración de Recursos Educativos. Formacion Universitaria, 6(5), 8.

Quintero, J. S. (2003). Estrategia integral para PyMES Innovadoras. Revista Escuela de Administración de Negocios(47), 12.

Rashida, A. M., Hanie, A. I., R. R., Fazlee, S. M., Abdul, K. N., Hassan , B. H., . . . Khairulnizam , Z. M. (2016). The Importance of Perceived Leadership Elements in Strategic Planning at Public University. In Procedia Economics and Finance, 39, 422-426.

Reguant-Álvarez, M., \& Torrado-Fonseca, M. . (2016). El método Delphi. REIRE,Revista d'Innovació $i$ Recerca en Educació, 87102. 
Tangarife Tellez, L., Sánchez Delgado, M., \& Rojas Contreras, W. (2014). Modelo de interventoría de tecnologías de información en el área de conocimiento de la gestión del alcance de pmbok ${ }^{\circledR} \mathrm{y}$ alineado con iso 21500 y cobit $\AA$. REVISTA COLOMBIANA DE TECNOLOGÍAS DE AVANZADA, $1(23)$.

Rochetti, J. L. (2006). An Integrated Balanced Scorecard Strategic Planning Model for. Journal of Practical Consulting, 25-35.

Rodríguez Valencia, J. (2016). Cómo aplicar la planeación estratégica a la pequeña y mediana empresas (6a. ed.). Distrito Federal, México: Cengage Learning.

Rojas López, Miguel David Medina Marín, \& Laura Joha. (2011). Planeación estratégica: fundamentos y casos. 126. Bogota D.C: Ediciones de la U.

Rytoft, C. (2013). Centros de datos. ABB Review, 84.

S. A. A. Acevedo, D. R. Bautista. (2017). Análisis de una red en un entorno IPV6: una mirada desde las intrusiones de red y el modelo TCP/IP. REVISTA COLOMBIANA DE TECNOLOGÍAS DE AVANZADA, ISSN: 1692-7257. 1(29).

Sanguino, M., Lobo, C., \& Arrieta, M. (2015). Diseño de un Plan Estratégico de Tecnologías de la Informacion para la Universidad Francisco de Paula Santander Ocaña. Ocaña.

Sistema Nacional de Informacion de la Educacion Superior. (2016). Matriculados Informacion 2016. Recuperado el 29 de 09 de 2017, de http://www.mineducacion.gov.co/sistemas deinformacion/1735/w3-article212400.html

Steiner, G. (2007). Planeación estratégica lo que todo director debe saber. Mexico: Grupo editorial patria.

Stern, N. (2002). A Strategy for Development. Washintong DC: Library of Congress Cataloging.

Tamayo, M. (2007). El Proceso de la Investigación Científica. Mexico: Noriega Editores.

Tamayo, M. (2012). El Proceso de la Investigación Científica (Cuarta ed.). México: Limusa.

Universidad de Pamplona. (2012). Plan de Desarrollo Institucional 2012-2020. Pamplona.

Universidad de Santander UDES. (2013). Plan de Desarrollo (2013-2018). Bucaramanga.
Universidad Francisco de Paula Santander. (2011). Plan de Desarrollo 2011-2019. Cucuta: Oficina de Planeacion Ufps.

Universidad Nacional de Colombia. (2016). Actualización Plan Estratégico de Tecnología. Bogotá. D.C.

Universidad Simon Bolivar. (2013). Plan Estratégico de Desarrollo (2013-2017). Barranquilla.

University, H. (2015). CIO Council Updated IT Strategic Plan. Cambridge, Massachusetts, EE. UU. : Harvard University.

Yujie, N., \& Wang, X. (2010). Research on the matching of IT strategic planning and business strategy. Chengdu, China: IEEE. 